\title{
Active control of the transmission of Lamb waves through an elastic metamaterial
}

\author{
W. Wang ${ }^{1}$, B. Bonello ${ }^{1, *}$, B. Djafari-Rouhani ${ }^{2}$, X. Fang ${ }^{3}$, Y. Pennec ${ }^{2}$, J. Zhao ${ }^{4}$, and Y. Jin ${ }^{4}$ \\ ${ }^{1}$ Sorbonne Université, UPMC Univ Paris 06 (INSP UMR CNRS 7588), 4 place Jussieu 75005 Paris, France \\ ${ }^{2}$ Université de Lille, IEMN UMR CNRS 8520, Département de Physique, Cité Scientifique, \\ 59652 Villeneuve d'Ascq cedex, France \\ ${ }^{3}$ Laboratory of Science and Technology on Integrated Logistics Support, \\ National University of Defense Technology, Changsha, Hunan 410073, China. \\ ${ }^{4}$ School of Aerospace Engineering and Applied Mechanics, Tongji University, \\ 100 Zhangwu Road, 200092 Shanghai, China
}

\begin{abstract}
We have numerically investigated the transmission of an antisymmetric Lamb wave through a line of silicon pillars erected onto a homogeneous silicon plate when the frequency is tuned to a resonant frequency of the pillars. For either a bending mode or a compressional mode, the resonators emit in the plate a wave $180^{\circ}$ out-of-phase with the exciting Lamb wave, resulting in dips in the transmission spectrum. We show that the transmission at resonance can be actively controlled by applying an external force, either tangential (bending eigenmode) or axial (compressional eigenmode), on top of the pillars. The transmission coefficient can be precisely controlled by finely tuning either the phase or the amplitude of the external force. For specific dimensions of the structure, both resonant modes arise at the same frequency. This geometry has the advantage of offering an additional degree of freedom for the direction of the external force but is less favorable from an energy point of view.
\end{abstract}

\section{INTRODUCTION}

Elastic metamaterials are dynamic structures made up of local resonators with lateral sizes much smaller than the wavelength of the elastic wave, embedded in a background or arranged on a free surface. The interest of the scientific community for these systems has known a continuous growth since they were proposed twenty years ago ${ }^{1}$ and the great deal of research that ensued, ${ }^{2-7}$ have significantly contributed to the possibility of controlling the propagation and the dispersion of acoustic/elastic waves. The abnormal effective properties of these artificial structures, namely the mass density and the compressibility that both may turn negative in certain frequency bands, have been successfully exploited in exotic phenomena including the subwavelength focusing of elastic energy, ${ }^{8,9}$ super airborne absorption in the audible spectrum, ${ }^{10,11}$ and wave guiding. ${ }^{12}$ Although the periodicity is not a requirement for achieving either of these negative properties, the resonators are generally regularly arranged. ${ }^{1,13-17}$ Bragg diffraction may therefore occur when the wavelength is of the order of the spacing between the inclusions, giving rise to frequency bands where the elastic wave propagation is prohibited. However, in the context of waves manipulation the most interesting property of these artificial structures is the occurrence of flat bands in their dispersion curves at much lower frequencies as compared to the Bragg band gaps. These relate to internal resonances and correspond to mechanical vibrations localized therein and in the immediate vicinity of the inclusions. Actually, immediately after investigations on elastic metamaterials have emerged it has been shown that the effective compressibility (resp. the effective mass density) becomes negative when the resonators are in a monopolar (resp. dipolar) motion, out-of-phase with respect to the waves in the background. ${ }^{1,2,18}$ Therefore, optimal control over the propagation of the acoustic/elastic waves must necessary include the control over both the vibration amplitude and the phase with respect to the motion in the background. From a practical point of view, such a control requires geometries allowing for the easy tuning of the motion of the resonators through an external field. Several solutions have been proposed in that respect. They are generally based onto the use of piezoelectric elements, ${ }^{19-21}$ static electric field, ${ }^{22}$ or electromagnets ${ }^{23}$ to tune the effective material parameters. They are effective at low or moderate frequencies and concern acoustic waves in a fluid as well as elastic waves in entirely solid metamaterials and metasurfaces. These structures are very promising for many

*Corresponding author: bernard.bonello@insp.jussieu.fr 
applications including control of elastic rays, ${ }^{24}$ sub-wavelength or adaptive focusing, ${ }^{8,25,26}$ energy harvesting, ${ }^{27}$ abnormal refraction of sound, ${ }^{28}$ topological phase transition and guiding, ${ }^{29-32}$ and structural health monitoring. ${ }^{33}$ Different structures have been proposed among which pillar-type acoustic metasurfaces have proven to be particularly interesting. Basically, these are periodic arrays of cylindrical pillars erected on one side or on both sides of a thin and homogeneous slab. This geometry is particularly attractive for tailoring the propagation of elastic waves in the plate since a pillar exhibits compressional and bending resonances that have respectively monopolar and dipolar symmetries. Both resonant frequencies can be independently tuned through a proper choice of the height and/or the diameter of the pillar that generally remains much less than the wavelength of the wave in the plate at resonance. Single-negative behavior can thus be obtained on purpose by a smart choice of the dimensions. Interestingly, both a compressional and a bending mode may arise at the same frequency for specific geometries of the pillars and the plate, ${ }^{34}$ and therefore these structures can potentially exhibit a double-negative behavior as well. In the linear regime, the displacement field behind a line of resonating pillars impinged by a Lamb wave can be regarded as the sum of the incident wave and a wave emitted by the set of resonators. It is expected that the transmission spectrum is different if the bending and the compression occur at the same frequency or not. When these two modes occur at different frequencies, the total transmitted amplitude is almost null for both. This can be ascribed to the interferences between incident and $\pi$ shifted emitted waves, both having comparable amplitudes. In contrast, the amplitude of the emitted wave significantly increases when the geometry of the structure is such that both bending and compressional modes occur at the same frequency. In that case, even though there is still a $\pi$ phase shift between incident and emitted waves, transmission of a substantial part of the elastic energy becomes possible.

We go a step further with the present work. Using numerical simulations based on a finite element method (FEM), we have investigated the transmission of the zero order antisymmetric Lamb wave through a line of pillars at resonance when an external force is applied on their top. We show that the transmission can be controlled at will by tuning both the amplitude and the phase of the applied force. When both the compressional and the bending modes of the resonator occur at the same frequency, an additional degree of freedom comes from the direction of the applied force that can be either parallel or normal to the pillar axis. We discuss the most appropriate choice in terms of the ratio energy cost/efficiency.

\section{EIGENMODES OF THE PILLARS AND EMITTED WAVES}

A finite element method was used to calculate the eigenmodes of a pillar on a plate. The method is based on the computing of the amplitude of the transmitted zero-order antisymmetric Lamb mode in the far field as a function of the frequency after the wave have traversed an infinite line of cylindrical pillars evenly spaced on a plate. The results were then normalized to the amplitude of the incident wave generated by a line-shaped harmonic source, parallel to the line of resonators and located a few hundred micrometers in front of it. Both the pillars and the plate were made of silicon and their dimensions were set in such a way that the mechanical resonances occur in the $\mathrm{MHz}$ range. The thickness of the plate, the height, and the diameter of the pillars were $t=145, h=245$ and $d=50 \mu \mathrm{m}$ respectively. The pillars were separated from each other by the period $a=200 \mu \mathrm{m}$, large enough to ensure that the coupling between the vibrations of neighboring pillars through the plate is weak and does not significantly alter the eigenfrequencies of the isolated resonator. In the frequency range of interest for this work, two sharp dips appear in the amplitude spectrum drawn as a black line in Fig. 1. At these frequencies, the amplitude of the transmitted wave is 0.38 and 0.08 respectively.

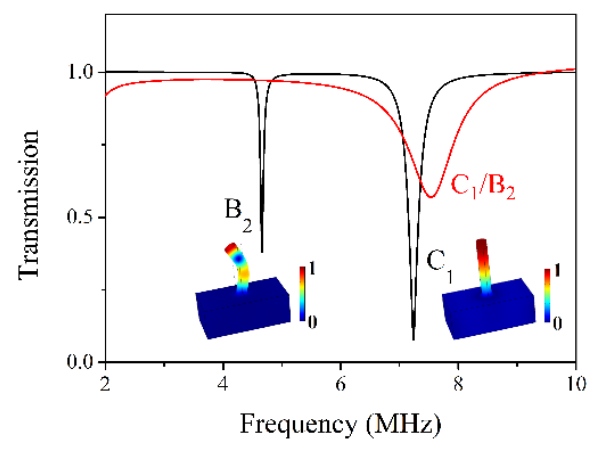

FIG. 1. Transmission through a line of pillars for which the first order compressional mode $\mathrm{C}_{1}$ and second order bending mode $\mathrm{B}_{2}$ arise at different frequencies ( $d=50 \mu \mathrm{m}$ - black line) or at the same frequency $(d=112 \mu \mathrm{m}-$ red line). The insets show the corresponding displacement fields.

They both correspond to a resonant mode which can be identified by the associated displacement 
field, also shown in Fig. 1, namely the second order bending mode, referred to hereafter as $\mathrm{B}_{2}$ and the first order compressional resonance denoted $\mathrm{C}_{1}$ that occur at $v_{\mathrm{B}_{2}}=4.66$ and $v_{\mathrm{C}_{1}}=7.24 \mathrm{MHz}$, respectively. In contrast to the mode $\mathrm{C}_{1}$ that arises at almost the same frequency upon a change in the diameter, the resonant frequency $v_{\mathrm{B}_{2}}$ is very sensitive to this parameter and increases as $d$ increases. ${ }^{34,35}$ Interestingly, for specific values of the diameter $d$, the modes $\mathrm{B}_{2}$ and $\mathrm{C}_{1}$ arise at the same frequency (see the red line in Fig. 1) giving rise to complex motions of the pillar. This behavior is observed at $v_{\mathrm{C}_{1} / \mathrm{B}_{2}}=7.57 \mathrm{MHz}$ when $d=112 \mu \mathrm{m}$ and all other dimensions kept unchanged. However, this motion can still be decomposed into compression and bending which can be independently monitored by calculating respectively the halfsum and the half-difference of the out-of-plane displacements measured at two diametrically opposite points on top of the pillar. Furthermore, the pillars and the plate being all made of the same material, it is expected that the vibrations of the formers strongly couple to the motion of the latter giving rise to a complex displacement field when a Lamb wave at a resonance frequency of the pillars passes through the structure.

When vibrating at resonance, each pillar in the line reemits a wave having the same symmetry as the exciting Lamb mode. ${ }^{16,34}$ In the linear regime where only small amplitudes are involved the superposition principle applies and the displacement field downstream of the line of pillars can be regarded as the sum of the incident and emitted waves. Therefore, subtracting the former from the total transmitted wave allows to thoroughly analyze the complex amplitude of the latter. The result is shown in Fig. 2(a) as a red line. This plot, computed at a point located $500 \mu \mathrm{m}$ behind the line of pillars, reveals the main features of the waves emitted around the two resonances $C_{1}$ and $\mathrm{B}_{2}$ when they occur at distinct frequencies. It corresponds to the complex amplitude of the emitted wave as a function of frequency of the wave emitted by an infinite line of pillars after they have been impinged at normal incidence by a zero order antisymmetric Lamb mode. In this computation, the origin of the phases is taken on the line source. In addition, it can be deduced from the variation of the real part of the wave emitted as a function of the frequency [blue line in Figure 2 (a)] that the modulus takes the values of 0.92 at the resonance frequency $v_{\mathrm{C}_{1}}=7.24 \mathrm{MHz}$ and
0.62 at $v_{\mathrm{B}_{2}}=4.66 \mathrm{MHz}$, which in both cases is the complementary value to 1 of the total transmitted amplitude [(see the black line in Fig. 1(a)]. This unambiguously shows that the transmission at resonance must be viewed as the superposition of two waves out-of-phase with each other.
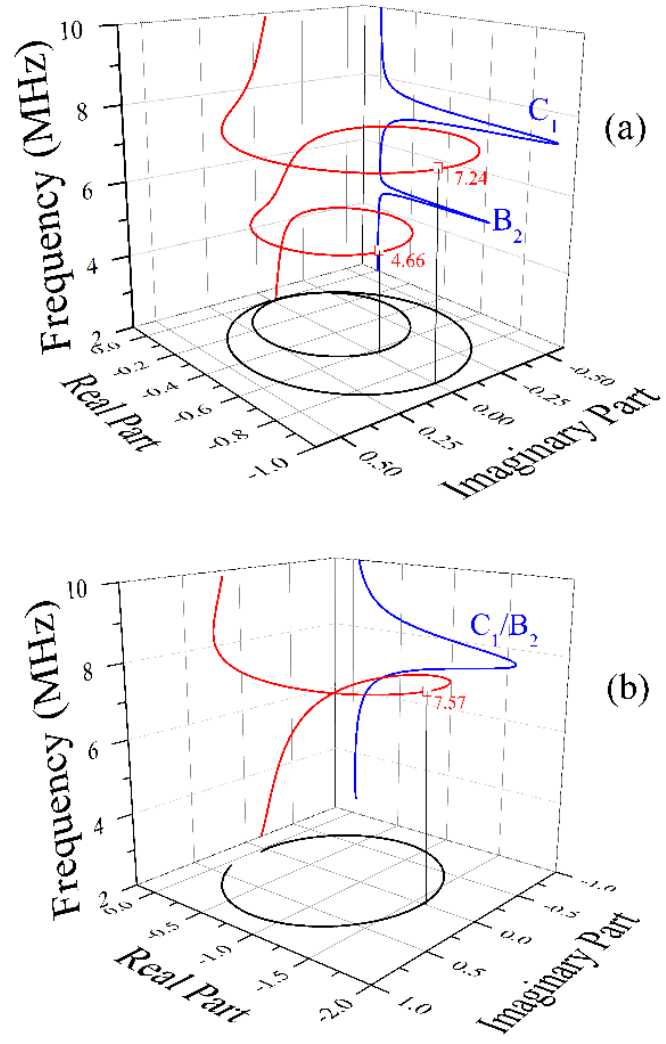

FIG. 2. (a) Complex amplitude of the antisymmetric Lamb wave emitted by a line of pillars as a function of the frequency when both resonances arise at different frequencies (red line). The dimensions are (in $\mu \mathrm{m}$ ) $t=145, h=245, d=50$, and $a=200$. The black lines correspond to the projection of the previous curve in the complex plane (Nyquist plot). The blue line is the real part of the emitted wave as a function of the frequency. (b) Same meaning as in (a) when both resonances arise at a single frequency. The diameter of the pillars is then $d=112 \mu \mathrm{m}$ and other dimensions kept unchanged.

Similar behavior is observed when the dimensions of the pillars are set so that the compression and the bending resonances occur at the shared frequency value $v_{\mathrm{C}_{1} / \mathrm{B}_{2}}=7.57 \mathrm{MHz}$, with nevertheless a difference in the maximum value of the amplitude of the complex emitted Lamb wave which goes to 1.5 in that case [blue and black lines in Fig. 2(b)]. This shows that the line of pillars is now partially transparent to the incident wave. It should be noted that in this work, we do not take into account the dissipative effects which only become important at high frequency since, in the 
linear regime, the damping is proportional to the frequency squared. The elastic energy which is not transmitted is therefore reflected.

\section{EXTERNAL CONTROL OF THE TRANSMISSION}

The transmission of the Lamb wave through the line of resonating pillars results from an interference mechanism between incident and emitted waves. Therefore, one should be able to tune as needed the transmission level by acting on either the amplitude or the phase of the interfering waves. The most suitable way of doing this, is to apply an external harmonic force on top of the pillars to force the out-coming wave to have the desired features. The layout we have considered for that is shown in Fig. 3(a). It consists of an infinite line of pillars aligned along the $x$ direction on a plate, as described in the preceding section. Antisymmetric Lamb waves excited by a source located $1000 \mu \mathrm{m}$ upstream of the line of pillars propagate in the plate. Perfect match layers (PML) are set on both ends of the unit cell to avoid any reflection from the edges. Depending on the symmetry of the resonant mode to be controlled, either a traction force along the $y$ direction at the frequency $v_{\mathrm{B}_{2}}$, or an axial force along the $z$ direction at $v_{\mathrm{C}_{1}}$, is applied on top of the pillars. In the following, we consider successively the cases where the two resonances arise at different frequencies and where they are simultaneously excited at a single frequency, i.e. $v_{\mathrm{B}_{2}}=v_{\mathrm{C}_{1}}$.

\section{A. Bending and compressional modes at distinct frequencies}

\section{A.1. Transmission at the frequency of the bending mode $\mathbf{B}_{2}$}

We define the transmission coefficient of $\mathrm{A}_{0} \mathrm{Lamb}$ mode $T_{\mathrm{A}_{0}}$ as the ratio of the out-of-plane displacement amplitude measured on the plate at a distance of $500 \mu \mathrm{m}$ downstream of the line of pillars, denoted "Detection" in Fig. 3(a) to the one measured at the same distance from the source, but on the plate free of pillars. The digital experiment consists in applying on top of each pillar in the line the harmonic traction force $F=F_{0} \sin \left(2 \pi v_{\mathrm{B}_{2}} t+\varphi\right)$, where $F_{0}$ and $\varphi$ are the amplitude per unit area and the phase respectively, and measuring the transmission coefficient as a function of both the independent parameters $F_{0}$ and $\varphi$. We set the maximum value of $F_{0}$ at $1 \mathrm{MPa}$, which corresponds to a load less than one gram for the pillars with the dimensions we consider here. This is a realistic value, easily achievable with several experimental techniques, including piezoelectric, magnetic, and even optical methods. As for the phase, it varies in between 0 and $2 \pi$.
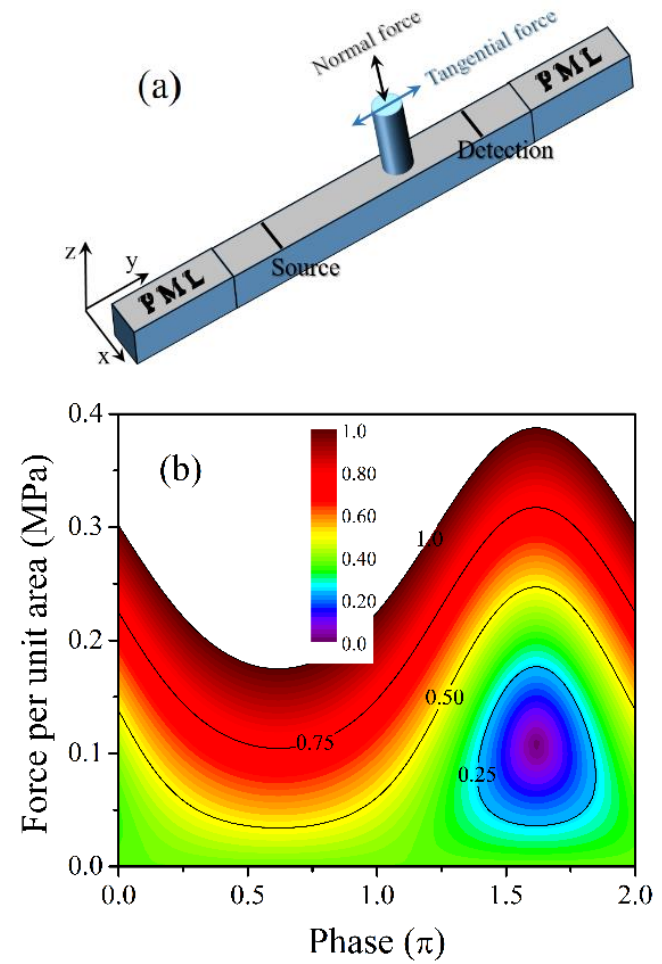

FIG. 3. (a) Finite elements model used to compute the transmission through an infinite line of pillars spaced $200 \mu \mathrm{m}$ along the $x$ direction. The source and detection are located at $1000 \mu \mathrm{m}$ and $500 \mu \mathrm{m}$ from the pillars respectively. (b) Transmission coefficient of zero order antisymmetric Lamb mode against the amplitude and the phase of a tangential harmonic force directed along the $y$ direction. The frequency is tuned to the bending resonance $v_{\mathrm{B}_{2}}=4.66 \mathrm{MHz}$. The white area corresponds to values of the transmission coefficient greater than 1 .

The transmission coefficient of $\mathrm{A}_{0}$ Lamb mode through the line of pillars against both parameters is displayed as a color-fill map in Fig. 3(b). The system being not isolated, $T_{\mathrm{A}_{0}}$ can take values greater than one; however only the values less than unity are shown in this figure. The main point to note in this result is that any value in between 0 and 1 is achievable for $T_{\mathrm{A}_{0}}$, showing that the structure allows to tune the transmission as needed or even to serve as a switch between the states "on" and "off". Although the condition to have zero transmission remains limited to a narrow range around $F_{0}=0.11 \mathrm{MPa}$ and $\varphi=1.62 \pi$, there is a large domain where $T_{\mathrm{A}_{0}}=1$ since this value can be obtained for any value of $\varphi$. The minimum force per unit area necessary for transmission 
equal to 1 is as small as $0.17 \mathrm{MPa}$; the required phase is then $\varphi \approx 0.62 \pi$. As expected from the data in Fig. 1, the transmission coefficient is 0.38 if $F_{0}=0$. Another point to be noticed is that $T_{\mathrm{A}_{0}}$ varies linearly as a function of the phase and the force per unit area up to $\sim 0.5 \mathrm{MPa}$, as evidenced both in Fig. 4(a) where we have drawn $T_{\mathrm{A}_{0}}(0.1, \varphi)$ as a black line and in Fig. 4(b) where $T_{\mathrm{A}_{0}}\left(F_{0}, 1.62 \pi\right)$ is shown.
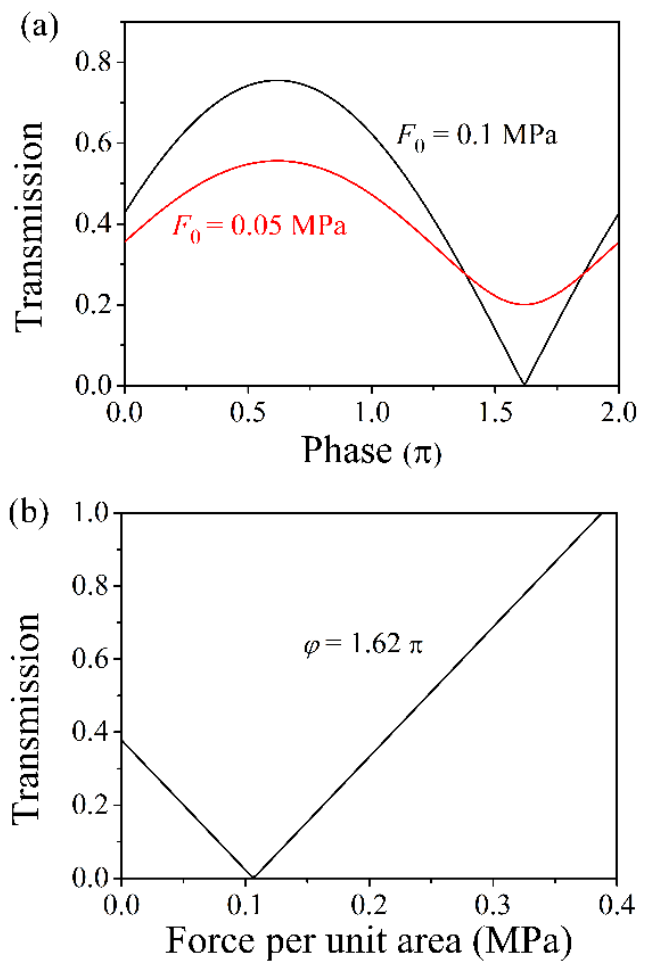

FIG. 4. (a) Transmission coefficient of $\mathrm{A}_{0}$ Lamb mode as a function of the phase of the tangential external force with an amplitude of $0.1 \mathrm{MPa}$ (black line) or $0.05 \mathrm{MPa}$ (red line) and (b) as a function of the amplitude when the phase is locked at $1.62 \pi$.

We show also in Fig. 4(a) as a red line the transmission coefficient when an external force of $0.05 \mathrm{MPa}$ is applied. As in the previous case, the maximum and minimum transmission arises when the phase is set to $\varphi=0.62 \pi$ and $\varphi=1.62 \pi$ respectively, suggesting that the displacement field downstream of the line of pillars should be interpreted in terms of interferences between the waves emitted by the source and by the pillars. Considering the phase relationship between both waves allows to further confirm this statement. Actually, if the origin of all phases being set at the source, the wave transmitted through the pillars and the one emitted by the pillars read at the detection point $T_{\mathrm{A}_{0}} \cos \left(2 \pi v_{\mathrm{B}_{2}} t+\varphi_{\mathrm{s}}\right)$ and
$F_{0} \cos \left(2 \pi v_{\mathrm{B}_{2}} t+\varphi+\varphi_{\mathrm{P}}\right) \quad$ respectively. Their respective phase at the detection point are $\varphi_{\mathrm{s}}$ and $\varphi+\varphi_{\mathrm{p}}$, where $\varphi_{\mathrm{s}}\left(\right.$ resp. $\varphi_{\mathrm{p}}$ ) relates to the delay time between the source (resp. the pillars) and the detection. The phase difference $\left(\varphi+\varphi_{\mathrm{P}}\right)-\varphi_{\mathrm{S}}$ against $\varphi$, computed in between $-\pi$ and $+\pi$ using the geometrical parameters summarized in Fig. 3(a) and displayed in Fig. 5, confirms that both waves are in-phase when $\varphi=0.62 \pi$, giving rise to constructive interferences, and out-of-phase when $\varphi=1.62 \pi$ where a minimum of transmission occurs.

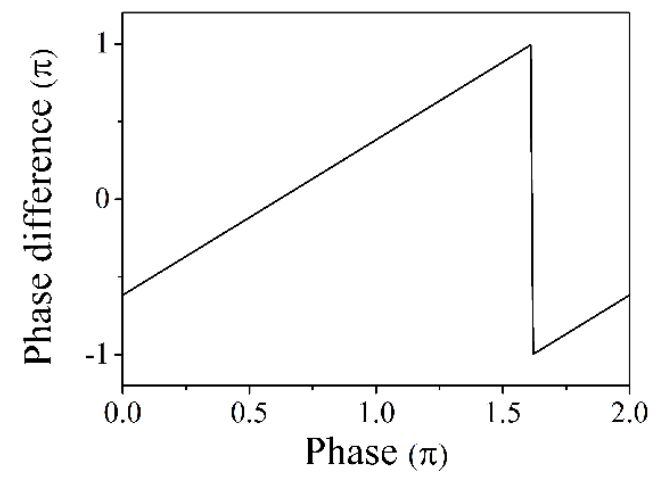

FIG. 5. Phase difference between the Lamb mode transmitted through the line of pillars and the wave emitted by the pillars upon excitation by an external tangential force.

It should be noticed that the displacement field associated to the bending resonance also features a component along the central axis of the pillars and therefore the external excitation of $\mathrm{B}_{2}$ could also be achieved owing to a harmonic force parallel to the $z$-direction. However, the amplitude $F_{0}$ necessary to observe the total transmission through the line of pillars would be as high as $6 \mathrm{MPa}$, instead of $0.17 \mathrm{MPa}$ with a tangential excitation, and almost $4 \mathrm{MPa}$ instead of $0.11 \mathrm{MPa}$ when the desired outcome is zero transmission. The energy cost is then too high and this approach is not suitable for controlling the propagation of the waves at the frequency of the bending resonance. Nonetheless, we show in the next section that applying a harmonic force along the axis of the pillars allows to finely tune the transmission at the frequency of the compressional resonance.

\section{A.2. Transmission at the frequency of the compressional mode $\mathrm{C}_{1}$}

We consider again the layout shown in Fig. 3(a) but now a force at the frequency of the 
compressional mode $v_{\mathrm{C}_{1}}=7.24 \mathrm{MHz}$ is applied normally on top of each pillar. Figure 6 where is displayed the transmission coefficient as a function of both the amplitude and the phase of the applied force, shows that even an amplitude less than $0.6 \mathrm{MPa}$ is sufficient to have the transmission coefficient continuously varying in between 0 and 1. The phase must then be locked at $1.17 \pi$. As previously, for any value of the phase there is an amplitude of the applied force allowing for total transmission of the antisymmetric Lamb mode, whereas the zero transmission remains restricted to a small domain around $F_{0}=0.04 \mathrm{MPa}$ and $\varphi=1.17 \pi$.

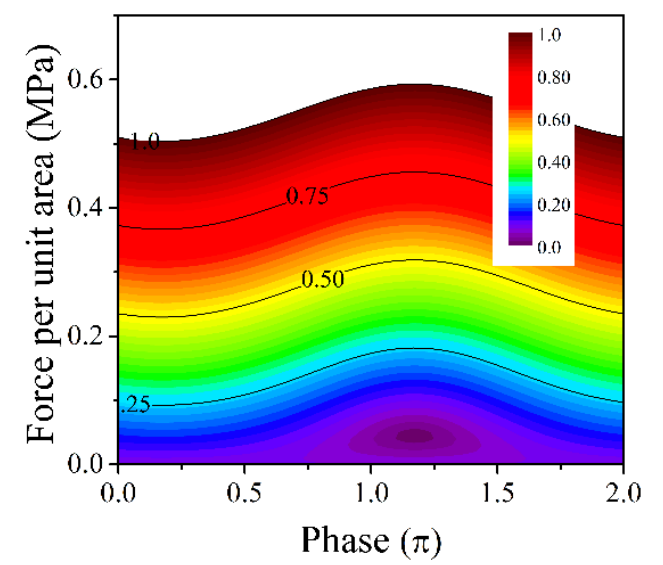

FIG. 6. Transmission coefficient of zero order antisymmetric Lamb mode against the amplitude and the phase of a compressional harmonic force directed along the $z$ direction. The frequency is tuned to a frequency close to the compressional resonance of the pillars $v_{\mathrm{C}_{1}}=7.24 \mathrm{MHz}$.

The dependence of the transmission through the line of resonators against the phase $\varphi$ of the applied force reflects its interferential origin. Actually, at any amplitude $F_{0}$, the transmission coefficient changes from minimum to maximum if an angle of $\pi$ is added to the phase $\varphi$. This is illustrated in Fig. 7 where we show in the upper panel the transmission coefficient as a function of $\varphi$ when $F_{0}=0.4 \mathrm{MPa}$ : the minimum $T_{\mathrm{A}_{0}}=0.65$ and maximum $T_{\mathrm{A}_{0}}=0.81$ transmission are for $\varphi=1.17 \pi$ and $\varphi=0.17 \pi$ respectively. In the lower panel in Fig. 7 we show the phase difference at the detection point, between the waves emitted by the source and by the resonating pillars, as a function of $\varphi$. When the transmission is minimum, both waves are out-of-phase giving rise to destructive interferences. The measured amplitude is then entirely due to the source and varies linearly with its amplitude. Similarly, maximum transmission occurs when both waves are in phase and constructively interfere.

\section{B. Bending and compressional modes at a single frequency}

The flexural modes of a pillar attached to a plate strongly depend on its diameter but are less sensitive to its height or to the thickness of the plate, in contrast to the compressional mode that can be slightly tuned through the value of the height but keeps almost the same frequency upon a change in the diameter or the thickness of the plate. ${ }^{34}$

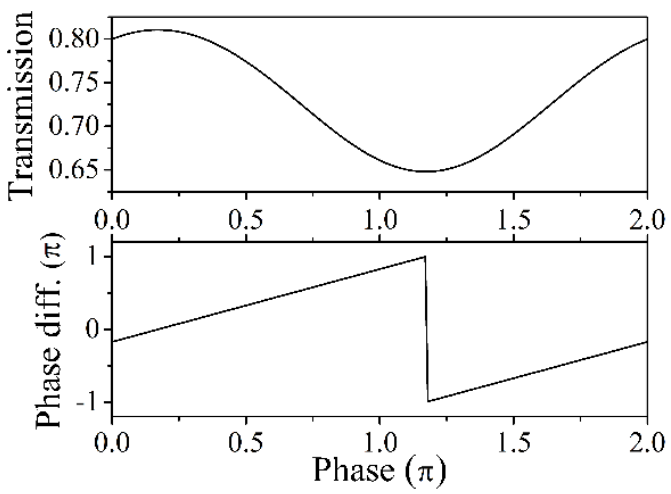

FIG. 7. Upper panel: Transmission coefficient for $\mathrm{A}_{0}$ mode against the phase of the applied force $\left(F_{0}=0.4 \mathrm{MPa}\right)$. Lower panel: Phase difference between the Lamb mode transmitted through the line of pillars and the wave emitted by the pillars upon excitation.

Thanks to these properties, it is possible to independently adjust the flexural and compressional resonances by the appropriate choice of geometric parameters and to make them appear both at the same frequency. A line of silicon pillars on a silicon plate having dimensions leading to the transmission spectrum represented by a red line in Fig. $1(d=112 \mu \mathrm{m}, h=245 \mu \mathrm{m}$, and $t=145 \mu \mathrm{m})$ fulfils this requirement. This composite resonance results in a dip rather than a peak in the transmission spectrum and does not allow zero transmission at the common frequency $v_{\mathrm{B}_{2} / \mathrm{C}_{1}}=7.57 \mathrm{MHz}$. We investigate in this section the effects of an external excitation at this frequency and we show that this type of resonance is nevertheless useful for controlling the transmission coefficient. This geometry has the advantage of offering an additional degree of freedom to control the transmission of the Lamb wave since the external excitation can be either 
tangential or axial, with however an efficiency that cannot be predicted a priori. The effect of a compressional excitation on the transmission at $7.57 \mathrm{MHz}$ is summarized in Fig. 8 (a). It is clear from this figure that a relatively low axial force makes it possible to finely control the transmission between 0 and 1 . The necessary amplitude to turn off the transmission of the wave is $F_{0}=0.28 \mathrm{MPa}$; the phase must then be adjusted to $\varphi=1.98 \pi$, whereas total transmission is achievable whatever is the phase, with a minimum amplitude of 0.21 $\mathrm{MPa}$; the phase must then be locked at $0.98 \pi$. We show for comparison the transmission coefficient map in Fig. 8(b) when a traction force is applied. Apart from the position on the phase axis of the null transmission that occurs now at $\varphi=1.42 \pi$, the dependences on $\varphi$ or $F_{0}$ are roughly the same. In particular, the minimum required amplitude of the applied tangential force to achieve total transmission is $F_{0}=0.18 \mathrm{MPa}$, i.e. $\sim 10 \%$ less than what is required with the external compressional force.
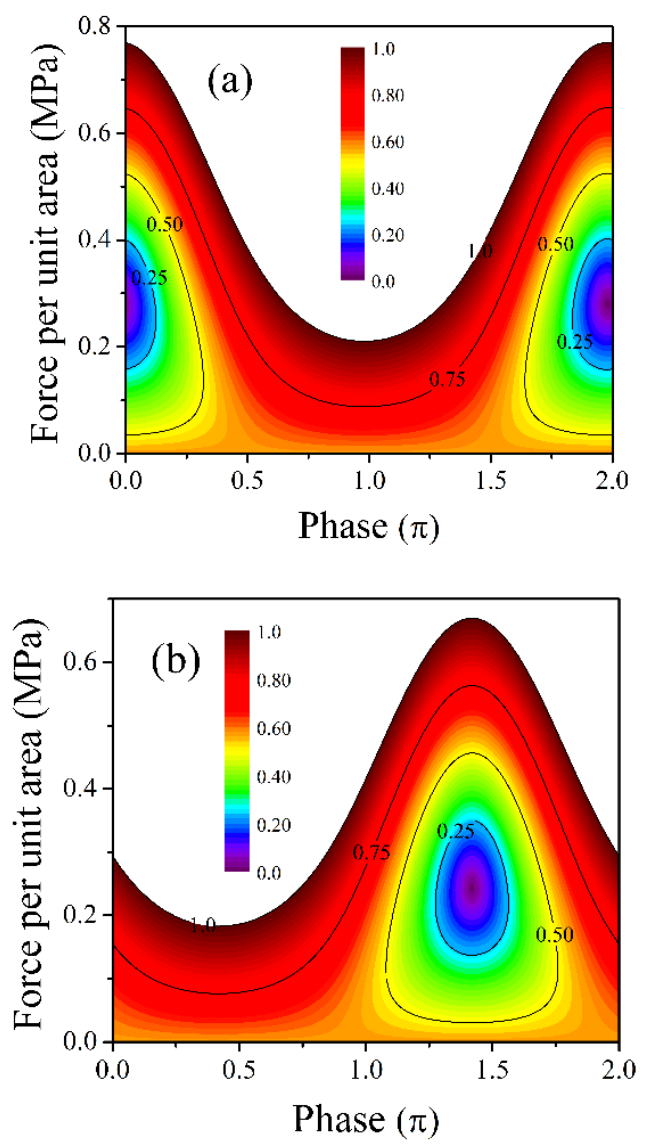

FIG. 8. (a) Transmission coefficient of zero order antisymmetric Lamb mode against the amplitude and the phase of a compressional harmonic force directed along the $z$ direction and (b) a traction force directed along the $x$ direction. In both cases, the frequency is tuned to a frequency close to the common frequency of the compressional and bending resonances of the pillars $v_{\mathrm{B}_{2} / \mathrm{C}_{1}}=7.57 \mathrm{MHz}$.

The phase relation between the wave emitted by the source and then transmitted behind the line of pillars and the wave resulting from the external excitation, computed at the detection point [see Fig. 3(a)], gives further evidence of the interferential nature of the displacement field downstream of the pillars. The phase difference between these waves, as a function of the amplitude of the applied traction force $F_{0}$, computed when the phase is set to $\varphi=1.42 \pi$, is drawn in the lower panel of Fig. 9; the transmission coefficient is shown in the upper panel. As long as $F_{0}$ is less than $0.25 \mathrm{MPa}$, increasing the amplitude of the applied force results in a decrease in the transmission coefficient, which is perfectly explained by the phase opposition between the waves generated by the source and emitted by the pillars, measured in this interval. Perfect cancellation accompanied by a $\pi$-phase jump occurs when $F_{0}=0.25 \mathrm{MPa}$. The linear increase in the transmission coefficient when $F_{0}$ is greater than $0.25 \mathrm{MPa}$ confirms that the two waves are then in phase and constructively interfere. Moreover, the phase difference being constant on both sides of the phase jump, the transmitted amplitude only depends on $F_{0}$.

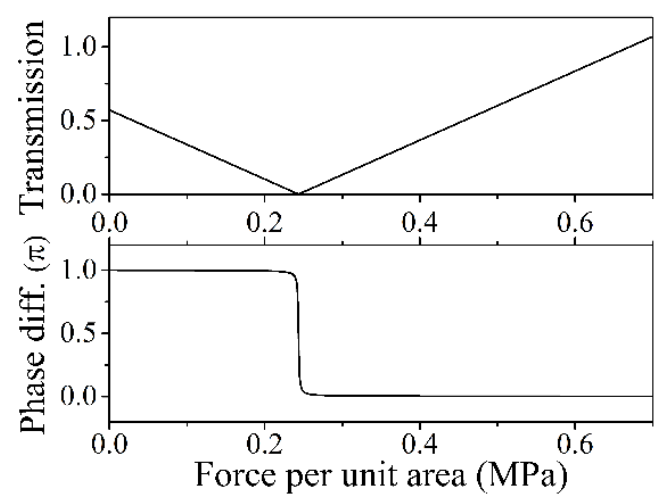

FIG. 9. Upper panel: Transmission coefficient for $A_{0}$ mode against the amplitude of the applied traction force at $v_{\mathrm{B}_{2} / \mathrm{C}_{1}}=7.57 \mathrm{MHz}(\varphi=1.42 \pi)$. Lower panel: Phase difference between the antisymmetric Lamb wave transmitted through the line of pillars and the wave emitted by the pillars.

\section{CONCLUSION}

With this work, we propose a method to adjust at will the transmission coefficient of an antisymmetric Lamb wave propagating through an infinite line of pillars. The method can be extended 
without difficulty to the case of the symmetric Lamb wave which also involves both in-plane and out-of-plane components, respectively sensitive to the bending and the compressional eigenmode of the pillars. Whether the eigenfrequencies are the same or distinct, the adjustment of the transmission coefficient in between 0 and 1 remains achievable by tuning, at constant phase, the amplitude of the external force; in all cases, the forces involved are weak and easy to implement. However, the necessary energy cost differs from one situation to another. Actually, when the eigenfrequencies are distinct, zero transmission is achieved at $v_{\mathrm{C}_{1}}=7.24 \mathrm{MHz}$ with an external compressional force per unit area of $\sim 0.04 \mathrm{MPa}$, which given the diameter of the pillar, corresponds to a force of $\sim 78 \mu \mathrm{N}$ only. More than three times higher traction force is required to achieve the same effect at the frequency of the bending mode $v_{\mathrm{B}_{2}}=4.66 \mathrm{MHz}$. It is the opposite situation to get $T_{\mathrm{A}_{0}}=1$ since the minimum necessary tangential force is then three times weaker than the minimum axial force $(330 \mu \mathrm{N}$ and $980 \mu \mathrm{N}$ respectively).

The implementation of a structure comprising pillars whose both normal modes occur at a single frequency has the advantage of offering an additional degree of freedom but is less favorable from an energy point of view. Indeed, a compressional force of about $2.8 \mathrm{mN}$ is then necessary to obtain $T_{\mathrm{A}_{0}}=0$. It is slightly less if using a bending force $(\sim 2.4 \mathrm{mN})$ but this remains thirty times higher than what must be used in the case of pillars with eigenmodes at distinct frequencies. Currently we are studying the extension of this work to the individual control of the pillars in the line in order to explore the functionalities of such a metasurface for various anomalous refraction phenomena and focusing effects. Indeed, by individually controlling each resonator, we can dynamically form a phase gradient distribution downstream of the line of pillars, in a similar way to what has been achieved recently statically using elastic phased diffraction gratings. ${ }^{36}$ By adjusting the phase of the force applied to each resonator, such a one-dimensional device would allow an incident wave to be redirected along any direction or to be focused at any point within a large area behind the pillars, in contrast to gradient index phononic lenses which, in general, only allow focusing along their median axis. ${ }^{8,25,27}$

Finally, we briefly discuss the experimental techniques that could be used to coherently control the resonators motions. Basically, this can be done in different ways, including piezoelectric, magnetic, and optical excitation of the pillars. Piezoelectric techniques allow for high strengths, high tunability, and operating frequencies in the $\mathrm{GHz}$ range, making such pillared structures compatible with silicon based MEMS if piezo transducers and electrodes are bounded both on top of the pillars and on the plate, but at the price of relatively delicate elaboration processes. To coherently control the vibration of the resonators and manipulate the propagation of waves at lower frequencies, the whole structure can be made of a piezoelectric material, as demonstrated recently with a lithium niobate platform. ${ }^{37}$ In that case, the FEM model must account for the coupling between elastic and electromagnetic waves, which entails simultaneously solving the equation of motion and Maxwell's equations. In the linear regime, this amounts to considering hardened elastic constants of the material, which may alter the phase relationship between transmitted and emitted waves. However, the general approach is unchanged and the main conclusions remains valid. On the other hand, when frequencies in the range of $\mathrm{kHz}$ and below are required, magnetic materials can be an easier alternative. They offer the additional advantage of being contactless allowing the application of the external force in any direction. Finally, all-optical techniques such as laser ultrasonics ${ }^{38}$ or picosecond time-domain thermoreflectance ${ }^{39}$ could establish the proof of concept of the active control of the transmission of Lamb waves with pillars based metasurfaces.

\section{AVAILABILITY OF DATA}

The data that support the findings of this study are available from the corresponding author upon reasonable request.

\section{ACKNOWLEDGEMENTS}

Wei Wang acknowledges the research scholarship No. 201608310130 provided by the China Scholarship Council. This work was partially supported by the National Natural Science Foundation of China (Grant No. 11902223)

\section{REFERENCES}

${ }^{1}$ Z. Liu, X. Zhang, Y. Mao, Y.Y. Zhu, Z. Yang, C.T. Chan, and P. Sheng, Science 289, 1734 (2000).

${ }^{2}$ J. Li and C. T. Chan, Phys. Rev. E (R), 70, 055602 (2004). 
${ }^{3}$ Y. Ding, Z. Liu, C. Qiu, and J. Shi, Phys. Rev. Lett. 99, 093904 (2007).

${ }^{4}$ S.H. Lee, C.M. Park, Y.M. Seo, Z.G. Wang, and C.K. Kim, Phys. Rev. Lett. 104, 054301 (2010).

${ }^{5}$ L. Fok and X. Zhang, Phys. Rev. B 83, 214304 (2011).

${ }^{6}$ C. Ding, L. Hao, and X. Zhao, J. Appl. Phys. 108, 074911(2010).

${ }^{7}$ Z. Liang, T. Feng, S. Lok, F. Liu, K. B. Ng, C.H. Chan, J. Wang, S. Han, S. Lee, and J. Li, Sci. Rep. 3, 1614 (2013).

${ }^{8}$ J. Zhao, B. Bonello, L. Becerra, O. Boyko, and R. Marchal, Appl. Phys. Lett. 108, 221905 (2016).

${ }^{9}$ X. Zhou, M.B. Assouar, and M. Oudich, Appl. Phys. Lett. 105, 233506 (2014).

${ }^{10}$ J. Mei, G. Ma, M. Yang, Z. Yang, W. Wen, and P. Sheng, Nat. Commun. 3, 756 (2012)

11 Z. Yang, H.M. Dai, N.H. Chan, G.C. Ma, and P. Sheng, Appl. Phys. Lett. 96, 041906 (2010).

${ }^{12}$ Y. Achaoui, A. Khelif, S. Benchabane, L. Robert, and V. Laude, Phys. Rev. B 83, 104201 (2011).

13 A. Bretagne, A. Tourin, and V. Leroy, Appl. Phys. 99, 221906 (2011).

14 Y. Pennec, B. Djafari-Rouhani, H. Larabi, J.O. Vasseur, and A.-C. Hladky-Hennion, Phys. Rev. B 78, 104105 (2008).

15 T. T. Wu, Z. G. Huang, T. C. Tsai, and T. C. Wu, Appl. Phys. Lett. 93, 111902 (2008).

${ }^{16}$ M. Oudich, B. Djafari-Rouhani, Y. Pennec, M.B. Assouar, and B. Bonello, J. Appl. Phys. 116, 184504 (2014)

17 T. Still, M. Oudich, G. K. Auerhammer, D. Vlassopoulos, B. Djafari-Rouhani, G. Fytas, and P. Sheng, Phys. Rev. B 88, 094102 (2013).

${ }^{18}$ T. Brunet, A. Merlin, B. Mascaro, K. Zimny, L. Leng, O. Poncelet, C. Aristegui, and O. Mondain-Monval, Nat. Mater. 14, 384 (2015).

${ }^{19}$ A. Baz, New J. Phys. 11, 123010 (2009).

${ }^{20}$ A. Baz, J. Appl. Phys. 125, 74503 (2017).

${ }^{21}$ B.I. Popa, L. Zigoneanu, and S.A. Cummer, Phys. Rev. B 88, 024303 (2013).

${ }^{22}$ S. Xiao, G. Ma, Y. Li, Z. Yang, and P. Sheng, Appl. Phys. Lett. 106, 091904 (2015).

${ }^{23}$ Z. Chen, C. Xue, L. Fan, and S.-Y. Zhang, X.-J. Li, H. Zhang, and J. Ding, Sci. Rep. 6, 30254 (2016).

${ }^{24}$ Y. Chen, X. Li, H. Nassar, G. Hu, and G. Huang, Smart Mater. Struct. 27, 115011 (2018).

${ }^{25}$ J. Zhao, B. Bonello, and O. Boyko, Phys. Rev. B 93, 174306 (2016).

${ }^{26}$ K. Yi, M. Collet, M. Ichchou, and L. Li, Smart Mater. Struct. 25, 075007 (2016).

${ }^{27}$ Y. Jin, D. Torrent, Y. Pennec, Y. Pan, and B. DjafariRouhani, J. Appl. Phys. 117, 244904 (2015).

${ }^{28} \mathrm{~S} . \mathrm{Li}, \mathrm{J} . \mathrm{Xu}$, and J. Tang, Appl. Phys. Lett. 112, 021903 (2018).

${ }^{29}$ W. Wang, Y. Jin, W. Wang, B. Bonello, B. DjafariRouhani, and R. Fleury, Phys. Rev. B 101, 024101 (2020).

${ }^{30}$ W. Wang, B. Bonello, B. Djafari-Rouhani, and Y. Pennec, Phys. Rev. B 100, 140101(R) (2019).
${ }^{31}$ W. Zhou, W. Chen, M. Destrade, C.W. Lim, Int. J. Mech. Sciences 180, 105668 (2020).

${ }^{32}$ W. Zhou, B. Wu, Z. Chen, W. Chen, C.W. Lim, J.N. Reddy, J. Mech. Phys. Sol. 137103824 (2020).

${ }^{3}$ C.T. Ng and M. Veidt, Smart Mater. Struct. 18, 074006 (2009).

${ }^{34}$ Y. Jin, B. Bonello, R.P. Moiseyenko, Y. Pennec, O. Boyko, and B. Djafari-Rouhani, Phys. Rev. B 96, 104311 (2017).

35 T.-T. Wu, Z.-G. Huang, T.-C. Tsai, and T.-C. Wu, Appl. Phys. Lett. 93, 111902 (2008).

${ }^{36}$ Z. Tian and L. Yu, Phys. Rev. Appl. 11, 024052 (2019).

37 L. Raguin, O. Gaiffe, R. Salut, J.-M. Cote, V. Soumann, V. Laude, A. Khelif, and S. Benchabane, Nat. Commun. 10, 4583 (2019).

38 R. Marchal, O. Boyko, B. Bonello, J. Zhao, L. Belliard, M. Oudich, Y. Pennec, and B. DjafariRouhani, Phys. Rev. B 86, 224302 (2012).

${ }^{39}$ B. Bonello, A. Ajinou, V. Richard, P. Djemia, and S.M. Chérif, J. Acoust. Soc. Am. 110, 1943 (2001). 